\title{
Heart rate variability biofeedback intero-nociceptive emotion exposure therapy for adverse childhood experiences [version
}

\section{1; peer review: 1 approved, 1 approved with reservations]}

\author{
Stéphanie Hahusseau1, Bruno Baracat ${ }^{2}$, Thierry Lebey ${ }^{3}$, Lionel Laudebat ${ }^{3}$, \\ Zarel Valdez ${ }^{3}$, Arnaud Delorme (iD-7 \\ ${ }^{1}$ Cabinet Médical de Stéphanie Hahusseau, Paris, France \\ ${ }^{2} \mathrm{EA}$ SCOTE, IU JF Champollion, Albi, France \\ ${ }^{3}$ LAPLACE, Federal University of Toulouse, Toulouse, France \\ ${ }^{4}$ CERCO, Universite Paul Sabatier, Toulouse, France \\ ${ }^{5}$ Centre de rechercher Cerveau et Cognition, CNRS, Toulouse, France \\ ${ }^{6}$ Institute of Neural Computation, University of California San Diego, Santa Diego, CA, USA \\ ${ }^{7}$ Institute of Noetic Sciences, Petaluma, CA, USA
}

V1 First published: 04 May 2020, 9:326
https://doi.org/10.12688/f1000research.20776.1

Latest published: 19 Apr 2022, 9:326

https://doi.org/10.12688/f1000research.20776.2

\section{Abstract}

Background: Psychiatric patients with adverse childhood experiences (ACE) tend to be dysfunctional in the interoceptive part of their emotional experience. The integration of interoceptive emotional activity in the insular and cingulate cortices is linked to the regulation of sympathovagal balance. This makes heart rate variability (HRV) an ideal measure for providing feedback on emotion regulation in real time.

Methods: A sample of one hundred $(n=100)$ outpatients was evaluated. Participants underwent eight 30-minutes ACE exposure sessions during which patients were guided to experience bodily sensations related to ACE while their HRV was monitored using a commercial biofeedback device.

Results: Comparing the results of first to last therapeutic session, a significant decrease in heart rate and an increase in HRV at the onset of the session were observed.

Conclusions: This study suggests physiological impact of therapeutic interventions on the autonomic balance and underlines the interest of $\mathrm{HRV}$ biofeedback as a clinical practice.

\section{Keywords}

Interoception, Adverse Childhood Experience, developmental trauma disorder, Biofeedback, Retrospective study, autonomic nervous system, PTSD

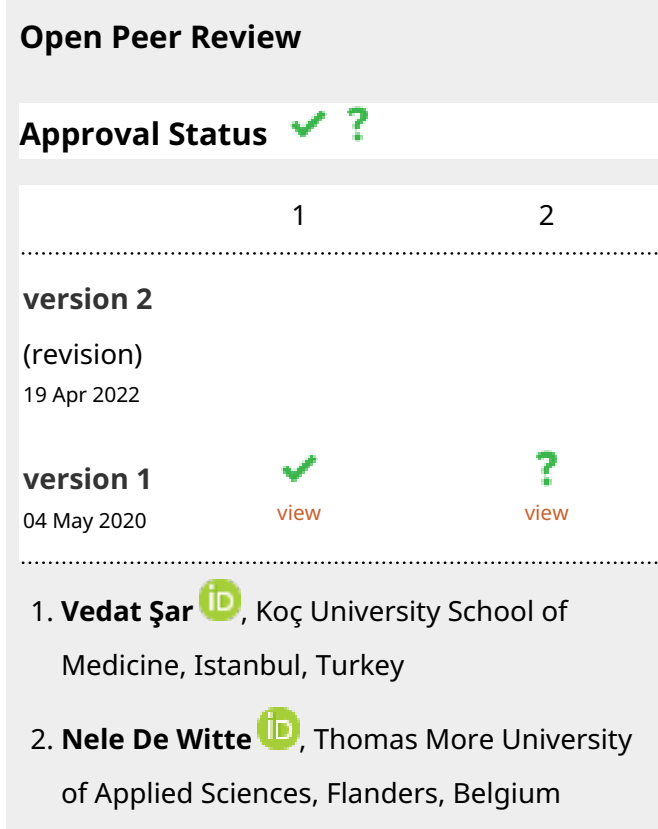

Any reports and responses or comments on the article can be found at the end of the article. 
Corresponding author: Arnaud Delorme (arnodelorme@gmail.com)

Author roles: Hahusseau S: Conceptualization, Data Curation, Investigation, Methodology; Baracat B: Resources; Lebey T: Supervision; Laudebat L: Software; Valdez Z: Software; Delorme A: Supervision

Competing interests: No competing interests were disclosed.

Grant information: The research was funded by the Conseil Régional de Midi-Pyrénée [n¹2052834]. The granting agency was not involved in the design of the study, the collection and analysis of the data, or in the writing and the submission of the present article. The funders had no role in study design, data collection and analysis, decision to publish, or preparation of the manuscript.

Copyright: @ 2020 Hahusseau S et al. This is an open access article distributed under the terms of the Creative Commons Attribution License, which permits unrestricted use, distribution, and reproduction in any medium, provided the original work is properly cited.

How to cite this article: Hahusseau S, Baracat B, Lebey T et al. Heart rate variability biofeedback intero-nociceptive emotion exposure therapy for adverse childhood experiences [version 1; peer review: 1 approved, 1 approved with reservations] F1000Research 2020, 9:326 https://doi.org/10.12688/f1000research.20776.1

First published: 04 May 2020, 9:326 https://doi.org/10.12688/f1000research.20776.1 


\section{Introduction}

Heart rate variability

Heart Rate Variability reflects an individual's ability to adaptively cope with stress. According to Thayer's model ${ }^{1}$ (see also 2,3), orthosympathetic activity is associated with higher central nervous system activity, in particular activity within the limbic system, the amygdala, and the prefrontal and frontal cortex ${ }^{4}$. One of the roles of these high-level structures is to inhibit the parasympathetic system and activate the sympathetic system. When a person faces a threat, this may elicit a hyperarousal and "flight or fight" response $\mathrm{e}^{5}$, which leads to an inhibition of the parasympathetic system and an activation of the sympathetic system. This corresponds to a decrease of HRV and often an increase in Heart Rhythm $(\mathrm{HR})^{6}$. Emotional events may have an influence on the general stress level$^{7}$, which in turn is visible in the sympathetic/ parasympathetic balance. Generally, these effects are transient because the higher nervous structures (essentially amygdala and prefrontal cortex) inhibit each other and, as soon as the stressor disappears, the system returns to parasympathetic tonus with low HR and high HRV.

For several decades, autonomic nervous system tests have been used to identify the physiologic correlates of psychiatric illnesses, particularly for affective and anxiety disorders ${ }^{8}$. In studies of Post-Traumatic Stress Disorder (PTSD) for example, decreased HRV are observed in PTSD patients compared to matched controls ${ }^{9}$. The HRV of PTSD patients indicates an increase in sympathetic activity and a reduction in parasympathetic activity. Patients suffering from PTSD tend to exhibit hyperactivity of the autonomous nervous system at rest and have been shown to be unable to further mobilize their orthosympathetic system when facing a stressful situation $^{10}$. In addition, the HRV profile after exposure to a trauma has been shown to be predictive of future traumatic episodes in PTSD $^{11,12}$. PTSD is associated with the disruption of the autonomic processes that maintain heartbeat regulation ${ }^{13}$.

\section{Clinical impact and assessment of adverse childhood experiences}

Research in psychiatry indicates that adverse childhood experiences leave durable physiological and neurophysiological traces and that there is a strong relationship between adverse childhood experiences and depression, suicide attempts, alcoholism, drug abuse, and other negative health outcomes ${ }^{14}$. Adverse childhood experiences (ACEs) are ubiquitous among the adult patient population ${ }^{15}$. The damaging effects of ACEs are nonspecific, thereby affecting a variety of functions and behaviors. In fact, ACEs have been shown to be negatively correlated with adult mental well-being ${ }^{16}$. Chronic traumatic experiences in childhood that extend over several years - as in cases with trauma and neglect - impair self-regulation function such as mood regulation and constancy in relations, described in "complex PTSD" and "developmental trauma disorder".

\section{Physiological impact of adverse childhood experiences}

Clinically, autonomic nervous system (ANS) function and emotional well-being are closely related ${ }^{17}$. Research has shown that having experienced early-life adverse events was associated with lasting effects on Heart Rate Variability (HRV) ${ }^{18}$, revealing complex interactions between traumatic experiences, ANS functioning and psychopathology ${ }^{19}$.

In addition, psychiatric research has shown that having experienced early-life adverse events was associated with altered interoception $^{20}$. Interoception is crucial for well-being ${ }^{21}$ as it mediates emotion regulation ${ }^{22}$. In fact, most psychiatric disorders are sustained by a type of interoceptive phobia ${ }^{23}$. Interoception require the interplay between perception of body states and cognitive appraisal of these states to inform emotional experience and motivating regulatory behavior ${ }^{24}$. The insular cortex in humans processes interoceptive activity and integrates and modulates cardiovascular, respiratory and emotional signals in order to create an integrated emotional experience $^{25}$.

\section{Evidence-based treatment for adults}

Neurophysiological impairments due to ACEs have been shown to be reversible ${ }^{26,27}$. Evidence-based psychotherapy for adults with ACE history typically involves a progression through three phases: safety and stabilization; trauma processing; consolidation of therapeutic gains ${ }^{28}$. The trauma processing phase requires sensitive therapeutic guidance. The other phases are best-practice approaches to all psychotherapeutic treatments, with the focus on the unique impact of ACEs.

Evidence based psychotherapy models for adults with ACEs-related disorders such as emotion-focused trauma therapy and eye-movement-desensitization-reprocessing are useful in the trauma processing phase. The efficacy of these approaches may be related to interoception rather than cognitive focusing ${ }^{29}$. Efficacy of psychotherapy with trauma patients may depend on the patient being able to face and feel adverse sensory and perceptual stimuli related to trauma-related memories in paced conditions $^{30}$.

Prolonged exposure therapy and cognitive processing therapy have gathered a significant amount of empirical support for PTSD treatment. However, they are not universally effective with patients continuing to struggle with residual post adverse childhood-traumatic symptoms. As such, other type of interventions such as biofeedback may be beneficial. When patients with PTSD were assigned to receive HRV biofeedback plus treatment, the results indicated that HRV biofeedback significantly increased the HRV while reducing symptoms of $\mathrm{PTSD}^{31}$. The present study intends to replicate these results using commercially available biofeedback equipment within an ecological therapeutic environment.

\section{Methods}

ACE therapy with interoceptive component

This procedure was developed by therapist MD SH (first author) for over more than 10 years. The therapeutic protocol comprises two parts. In the first part, which typically comprised eight weekly sessions of half an hour each, the therapist (co-author $\mathrm{SH}$ ) identified the occurrence of adverse childhood experiences 
(psychological abuse, physical abuse, contact sexual abuse, or exposure to household dysfunction during childhood, e.g. exposure to substance abuse, mental illness, violent treatment by parent or stepparent, criminal behavior in the household). To do so, the therapist carried out clinical investigations, collected anamnestic and diachronic data, and guided the patient to specific breathing visualization exercises.

In order to characterize adverse childhood memories responsible for interoceptive phobia, the patient was asked to initially focus his attention on his/her breath, then on nociceptive sensations, and finally on the childhood memories ${ }^{32}$. The therapist asked the patient to focus his/her attention on his/her breathing while describing the images associated with the memories and specific body sensation or pain that might arise in detail. This exercise was carried out with closed eyes. During this exercise each uncomfortable physical sensation and negative thought was rated in terms of intensity on a 10-point scale. Later, after a meeting devoted to the conceptualization of the selected traumatic memories and their influence on repetitive negative emotions, the therapist helped the patient to establish a coherent narrative within which to frame his/her difficulties. The practitioner explained the therapeutic hypothesis, which would be instantiated in the second phase of the therapy indicated as described below.

The second phase of the therapy consisted of bi-monthly one-hour therapeutic sessions. In each session, after five minutes of rest, the therapist asked the patient to wear an ear device sensor which is part of a HRV biofeedback device (Emwave2; Heartmath, Inc.). The patient was then asked to focus his/her attention on his/her breathing for two minutes. After two minutes, the evocation of images related to the adverse childhood memory chosen for this session started ${ }^{33}$. To avoid dissociative processes and develop interoception and parasympathetic activation, the patient was asked to focus his/her attention on the uncomfortable bodily sensations for about 30 minutes $^{34}$. Feedback on the sympathetic-vagal balance was directly affected by the sound delivered by the biofeedback device. The sound of the biofeedback device is correlated with the low frequency peak in the HRV spectrum (HeartMath Emwave 2 device and associated software; US patent 6,358,201 and Australian patent 770323). The number of sessions depended on the number of adverse childhood experiences to face - in general about 6 sessions. During these meetings, the therapist saved the series of heart beat intervals ( $\mathrm{R}$ to $\mathrm{R}$ intervals) using the biofeedback software. In this study, five minutes of data at the beginning and at the end of the first session of phase 2 (session 1) and the last session of phase 2 (designated as "session 2" even though there might be several sessions between "session 1" and "session 2") have been analyzed. Each of these 5 minutes comprise 2 minutes of breathing plus the evocation of traumatic imagery.

\section{Patient's inclusion}

The most recent 100 outpatients of therapist $\mathrm{SH}$ having experienced at least one type of adverse childhood experience and having used the biofeedback method described above were retained as study population. Only patients for whom more than 3 consecutive sessions were collected were included. These two conditions were the only inclusion criteria. 100 patients was judged appropriate for an HRV study of this nature based on the literature ${ }^{35,36}$. In general, HRV studies require about of 100 patients or subjects to observe links between mental condition and HRV measures, although some studies have observed significant effects in depressive subjects with group sizes as low as $27^{37}$. Inclusion criteria included an history of adverse childhood experience (therapist assessment). Patient who required psychiatric medical treatment (therapist assessment) were excluded from the study. Patients were included regardless of DSM V guidelines for trauma since these do not provide a definition for patients having experienced chronic trauma over several years such as neglect. However, sub-categories in the DSM V were considered as described in a later section. The data was collected over one year. Table 1 summarizes the main features of the data sample.

\section{Compliance with ethical standards}

The local ethical committee (Comité de protection des personnes Sud Ouest) approved the study and the use of the data for research purposes. Since the study was performed retrospectively, no patient consent was necessary. However, the French national entity for the protection of public and medical digital records (CNIL) authorized the retrospective use of the clinical data for this research (authorization number 1685185). The therapist associated a random number to each patient which was then used to anonymize the questionnaire data, the scanned notes of the therapist and the EKG files of each patient. Except for the therapist (co-author $\mathrm{SH}$ ), all other investigators were blind to the identity of the patients. The blinding procedure consisted in assigning a randomly generated code to patients, in compliance with CNIL requirements (Commission nationale de l'informatique et des libertés). It was performed at the therapist's office by the therapist herself to ensure that no identifiable document could inadvertently be lost, stolen, or read by anyone else than the therapist. When a paper form contained identifiable information, it was masked by the therapist, a sticker with the anonymized patient ID was temporarily placed on the form and the form was photocopied for later digital transfer. The questionnaire data was not integrated into the current report to focus on the interpretation of heart beat intervals.

\section{Data collection and data processing}

$\mathrm{R}$ to $\mathrm{R}$ intervals were collected during therapeutic sessions using the biofeedback Emwave2@ device. This system uses a photoplethysmographic sensor located on the right ear lobe

Table 1. Data sample statistics.

\begin{tabular}{|l|l|l|}
\hline & Men & Women \\
\hline Number of participants & 20 & 80 \\
\hline Mean age in years & 37.5 & 37.8 \\
\hline Mean number of session & 6.5 & 5.5 \\
\hline Mean duration of therapy (in days) & 206 & 201 \\
\hline
\end{tabular}


and series of heart beats are automatically extracted by the biofeedback software. The accuracy of this data was verified in one subject by comparison to a simultaneously recorded real EKG (Biopac MP36 unit and Acqknowledge $\subset$ using Einthoven Lead II derivation): the heart beats monitored by the biofeedback system were delayed in comparison to the EKG based on the time it takes for blood pressure to build up at the ear lobe. Except for this delay, heart beat measurements were accurate within millisecond precision in comparison to those visible on the EKG. Using heart beat time intervals over 300 seconds, HRV calculations were carried out with the Biomedical Toolkit used on Labview@ version 2009. This software performs HRV calculation in the same way as other HRV software packages do - such as the popular Kubios software (Kubios Oy, Finland). $\mathrm{R}$ to $\mathrm{R}$ intervals were resampled at $8 \mathrm{hz}$, and the power spectrum was calculated over the whole 5-minute record using an FFT decomposition. Power was obtained at each frequency by calculating the square value of the FFT absolute amplitude. In the frequency domain, total HRV was obtained by summing the total spectral power for the low frequency band (LF) $0.05 \mathrm{~Hz}-0.15 \mathrm{~Hz}$ and the high frequency band (HF) $0.15 \mathrm{~Hz}-0.35 \mathrm{~Hz}$. The $\mathrm{LF} / \mathrm{HF}$ ratio was also calculated. Before performing statistical analyses, a $\log$ (Ln) transformation was applied and values were subsequently normalized across subjects. Other heart measures calculated in the time domain were Heart Rate (RR), Root Mean Square of Standard Deviation of $\mathrm{R}$ to $\mathrm{R}$ intervals (RMSSD), proportion of $\mathrm{R}$ to $\mathrm{R}$ intervals larger than $50 \mathrm{msec}$ (pNN50), and Triangular Index of $\mathrm{R}$ to $\mathrm{R}$ intervals.

The clinical assessment of the therapist led to the creation of the following categories mapped onto DSM V categories: Substance abuse (SA); Somatoform Disorders (SD); Anxious Disorders (AD); Serious Personality Disorder (SPD); Post Traumatic Stress Syndrome (PTSS) (Data not included). All the patients could be diagnosed with trauma complex or developmental trauma disorder ${ }^{38}$. In addition to these categories, additional independent variables were retained: patient age, patient sex, the number of meetings in phase 2 , and the number of days between the first and the last data recording sessions. Data was collected by the therapist on custom forms (available as extended data $^{37}$ ) that were later transcribed into digital form after the anonymization process.

\section{Statistical procedure}

Changes in the HRV between the two selected therapy sessions and within each session between the beginning and the end of each recording were analyzed using 2-way repeated measure ANOVA. Measurements related to the first meeting of therapy of phase 2 are indicated by "session 1" and a measurement at the end of the session of phase 2 is indicated by "session 2". For each of these sessions, a measurement was taken at the beginning of the session (indicated by "Measurement 1") and another at the end of the session (indicated by "Measurement 2"). There is about 25 minutes delay between "Measurement 1 " and "Measurement 2" during which the patient was asked to re-experience traumatizing events - this time frame was not analyzed.
Statistical analyses combine two within-subjects factors with two levels; "Session" and "Measurement". Additionally, other between-subjects factors and independent variables described in the previous section were included. All the analyses were carried out with General Linear Model (GLM) module of SPSS(C) (version 17) by using the statistics of Greenhouse-Geisser.

The existence of corrupted $\mathrm{R}$ to $\mathrm{R}$ series and/or incomplete data associated with the statistical method used (within-subjects measurement) implies that the number of subjects included in the statistical analyses was lower than 100, and varies depending on the type of analysis. $\mathrm{R}$ to $\mathrm{R}$ and demographic data are available as underlying data ${ }^{37}$.

\section{Results}

Significant changes in HR and HRV were observed. HR was higher by 3.4 beat per minute (bpm) in session 1 compared to session 2 $(\mathrm{D}=4.99 ; \mathrm{DF}=1,55 ; \mathrm{p}=0.029)$. Within sessions HR increased by $1.6 \mathrm{bpm}(\mathrm{D}=23.53 ; \mathrm{DF}=1,55 ; \mathrm{p}<0.001)$. There was no interaction between these two factors.

Globally, total HRV estimated in the frequency domain showed significant changes as well. Within a session, HRV decrease was significant $(\mathrm{D}=10.97 ; \mathrm{DF}=1,55 ; \mathrm{p}=0.002)$. The total quantity of transformed HRV decreased by 0.245 points between the beginning and the end of the therapy but failed to reach significance. The interaction between the two factors was significant ( $D=13.32 ; \mathrm{DF}=1,55 ; \mathrm{p}=0.001$ ). This is due to the fact that of the decrease in HRV between Measurement 1 and Measurement 2 was relatively large during the second session $(0.476$ points; $\mathrm{DF}=1,55 ; \mathrm{p}<0.05)$, but relatively low for session $1(0.014$ points; ns). Table 2 summarizes mean HRV values and standard errors of the mean. Figure 1 summarizes the variations in HRV based on the two factors - the Z score of $\mathrm{Ln}(\mathrm{HRV})$ was plotted where the difference were most striking.

All other analyses of measurements of HRV obtained in the frequency domain ( $\mathrm{LF}, \mathrm{HF}, \mathrm{LF} / \mathrm{HF}$ ) or time domain did not lead to significant differences. Additional inclusion of factors ("Clinical Opinion", "Sex" as between subject factor, "Age", "Number of days between Session 1 and Session 2", "Mean number of meetings" and "Time between the two sessions" as covariates in between-subject factor) in the ANOVA did not lead to significant differences and did not modify the level of significance of the differences mentioned above. Table 3 shows the spectral LF and HF values for the different sessions and measurements.
Table 2. Mean heart rate variability (HRV) and standard error of the mean (in parenthesis) for all the sessions and measurements.

\begin{tabular}{|l|r|r|}
\hline & Measurement 1 & Measurement 2 \\
\hline Session 1 & $9834(1329)$ & $10907(1887)$ \\
\hline Session 2 & $7889(939)$ & $10602(1124)$ \\
\hline
\end{tabular}




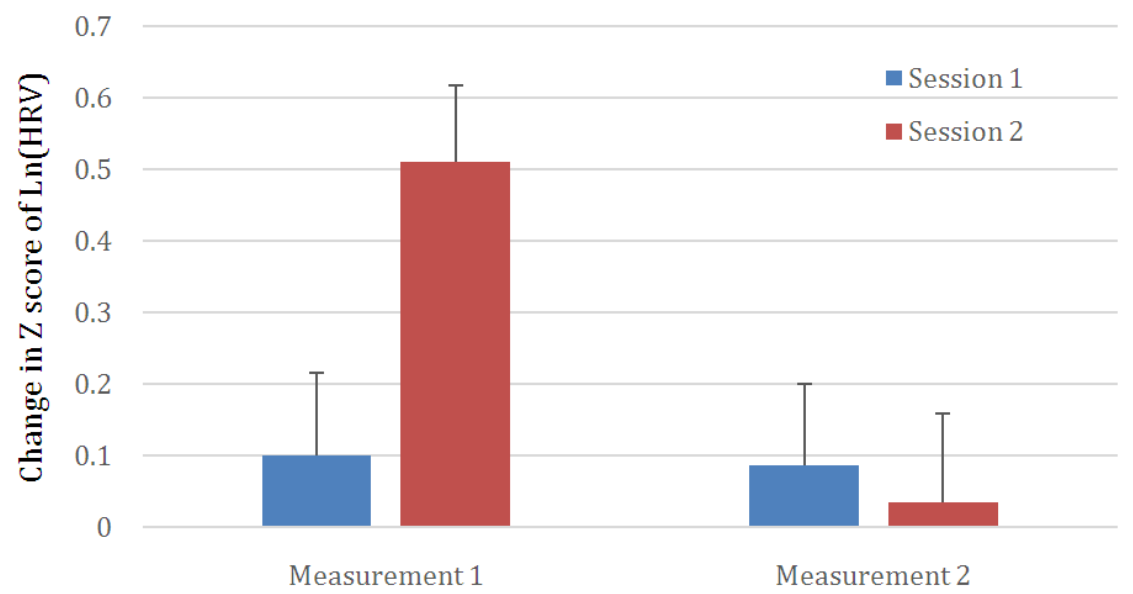

Figure 1. Changes in Z score of Ln(HRV (heart rate variability)) (Ln for Napierian Logarithm) for the first and last sessions ("session") and within session ("Measurement").

Table 3. Mean high frequency (HF) and low frequency (LF) spectral values and standard error of the mean (in parenthesis) in $\mathrm{ms}^{2} / \mathrm{hz}$ for all the sessions and measures.

\begin{tabular}{|l|r|r|l|r|r|}
\hline LF & Measurement 1 & Measurement 2 & HF & Measurement 1 & Measurement 2 \\
\hline Session 1 & $9637(1321)$ & $10694(1876)$ & Session 1 & $197(21)$ & $214(32)$ \\
\hline Session 2 & $10972(1102)$ & $10409(1117)$ & Session 2 & $231(23)$ & $194(18)$ \\
\hline
\end{tabular}

\section{Discussion}

The present study demonstrates an effect of biofeedback therapeutic interventions both in terms of heart rhythm and heart rhythm variability measurements. Subjects' HR showed a significant decrease between session 1 and session 2 which could indicate reduced chronic stress. The reduction in the average HR in session 2 compared to session 1 can be interpreted as an effect of therapeutic interventions.

Moreover the patient average HR increased between the beginning and the end of each of the two sessions. This increase in the HR is consistent with the model of Thayer ${ }^{17}$ : the patient experiences a change in emotional state due to the recall of the traumatic experience, and the induced stress leads to an increase in HR.

The analysis of the modifications of HRV partially confirms this interpretation. At the onset of session 2, patients had higher HRV than at the onset of session 1, which indicates larger parasympathetic influences towards the end of the therapy. Also, in the general population, HRV tends to be lower in patients compared to controls. In the task force of the European Society of Cardiology and the North American Society of Pacing Electrophysiology ${ }^{39}$, HRV of control subjects in decubitus dorsal at rest over 5 minutes were $3466 \mathrm{~ms}^{2} / \mathrm{hz}$ $\left( \pm 1018 \mathrm{~ms}^{2} / \mathrm{hz}\right)$. Measurements for the present study were approximately three times lower which could mean that HRV is close to its minimum. $1085 \mathrm{~ms}^{2} / \mathrm{hz}$ (standard error of the mean (s.e.) $1329 \mathrm{~ms}^{2} / \mathrm{hz}$ ) were calculated for "Session 1-Measurement
1", $1094 \mathrm{~ms}^{2} / \mathrm{hz}$ (s.e. $1887 \mathrm{~ms}^{2} / \mathrm{hz}$ ) for "Session 1-Measurement 2",1195 $\mathrm{ms}^{2} / \mathrm{hz}$ (s.e. $939 \mathrm{~ms}^{2} / \mathrm{hz}$ ) for "Session 2-Mesurement 1" and $1080 \mathrm{~ms}^{2} / \mathrm{hz}$ (s.e. $1123 \mathrm{~ms}^{2} / \mathrm{hz}$ ) of "Session 2-Measurement 2". A possible interpretation for the reduction in HRV within session 2 (and not within session 1), is that the HRV at the onset of session 2 was high enough to allow for a reduction associated with the emotional trauma recall. This was not the case in session 1 where the initial HRV was lower than in session 2 and thus might not have allowed for further reduction in HRV induced by trauma recall.

Differences in HRV total power but not in the Low Frequency (LF) and High Frequency (HF) of the HRV were observed. The absence of an effect on HF and LF across conditions could be explained by the important inter-individual variability. HF values were weak (average of all conditions $220 \mathrm{~ms}^{2} / \mathrm{hz}$ ) compared to LF values (average of all conditions $1123 \mathrm{~ms}^{2} / \mathrm{hz}$ ). This means that the major part of the total HRV power was due to the LF component and the LF coefficient of variation was large (ranging between 1.07 and 1.94). Finally, differences of HRV between sessions and measurement times, calculated at the individual level, ranged between $-1105 \mathrm{~ms}^{2} / \mathrm{hz}$ and $+1151 \mathrm{~ms}^{2} / \mathrm{hz}$ which means that irrespective of the comparison, there were almost as many patients whose HRV varies in one direction as ones whose HRV varies in the opposite direction.

The absence of a control group and the naturalistic conditions of this retrospective study, carried out with the constraints imposed 
by clinical out-patient medical practice, are not ideal, and resulted in large inter-individual measure variances. In this retrospective study, a large number of variables not included in the analysis might also have influenced outcome measures. For example, the decision to follow the therapy could have been accompanied by a change in lifestyle (i.e. general improvement of the hygiene of life), which may affect both HR and HRV measures. In addition, time alone could have been responsible for changes in HRV. As this study has been conceived a posteriori, such variables could not be controlled. However, the absence of statistical effects associated with biographical variables indicates that these types of effects are relatively unlikely.

The intra-individual differences in the emotional reactivity following the evocation of the traumatic memory were difficult to standardize. One possible solution could be the consideration of an individual cardiovascular reactivity, which may be modeled as influenced by several independent factors ${ }^{40}$. One of these factors would depend on individual physiological variables and be independent of the nature and the intensity of the emotional trauma evoked during therapy. This factor could be estimated separately using simple tests which have been used to establish relationships between the variations of HRV and the ability to regulate emotions ${ }^{6}$. Other factors, such as the intensity of the trauma and the type of trauma could also influence cardiac reactivity. This multi-factor type of modeling could potentially help to reduce and understand inter-subject variability and lead to HR and HRV measures with diagnostic and therapeutic value.

In this protocol which includes two therapeutic components; HRV biofeedback and intero-nociceptive exposure, it is impossible to distinguish the impact of one component versus the other. The hypothesis was that both components are important, and that it is the combination of the two which maximizes the therapeutic effect. Further studies will be necessary to investigate this hypothesis.

The analysis of HRV is a simple and noninvasive method to quantify the activity of the autonomous nervous system. The sympathetic-parasympathetic balance of patients having undergone important traumas is modified in favor of sympathetic influences. This study shows that interoception exposure therapy - combined with biofeedback - was able to increase parasympathetic influences. Furthermore, progressive reduction in the cardiac rhythm and an increase in HRV at rest over a period of a few months were demonstrated. It is important to note that these variations were independent of the disorder diagnosed by the Psychiatrist, therefore the HRV might be considered as a general indicator of health. These results warrant further investigation of both therapeutic components (HRV biofeedback and intero-nociceptive exposure) and their comparison to other types of interventions.

\section{Data availability}

Underlying data

Zenodo: R-R HRV data from Biofeedback on 100 patients. http://doi.org/10.5281/zenodo. $3703130^{37}$

This project contains the following underlying data:

- Archive_RR_All_subjects (folder containing R - R interval data for all participants as .txt files. Participants can be identified using the ID (e.g. n1799) in the file name)

- biographic_data.txt (Demographic data for participants)

\section{Extended data}

Zenodo: R-R HRV data from Biofeedback on 100 patients. http://doi.org/10.5281/zenodo.3703130 37

This project contains the following extended data:

- info_sheet.docx (Study data collection form, English)

- info_sheet_fr.docx (Study data collection form, French)

Data are available under the terms of the Creative Commons Attribution 4.0 International license (CC-BY 4.0).

\section{Acknowledgements}

The authors wish to thank The Research and Editing Consulting Program (RECP) for their editing services.
1. Thayer JF, Lane RD: Claude Bernard and the heart-brain connection: further elaboration of a model of neurovisceral integration. Neurosci Biobehav Rev. 2009; 33(2): 81-88.

PubMed Abstract | Publisher Full Text

2. Berston GG, Cacioppo JT, Grossman P: Whither vagal tone. Biol Psychol. 2007; 74(2): 295-300.

PubMed Abstract | Publisher Full Text

3. Gianaros, PJ, Sheu LK, Matthews KA, et al: Individual differences in stressorevoked blood pressure reactivity vary with activation, volume, and functiona connectivity of the amygdala. J Neurosci. 2008; 28(4): 990-999. PubMed Abstract | Publisher Full Text | Free Full Text

4. Buchanan TW, Driscoll D, Mowrer SM, et al.: Medial prefrontal cortex damage affects physiological and psychological stress responses differently in men and women. Psychoneuroendocrinology. 2010; 35(1): 56-66. PubMed Abstract | Publisher Full Text | Free Full Text

5. Cannon WB: Pharmacological injections and physiological inferences. Science. 1929; 70(1821): 500-1.

PubMed Abstract | Publisher Full Text

6. Kreibig SD: Autonomic nervous system activity in emotion: a review. Biol Psychol. 2010; 84(3): 394-421.

PubMed Abstract | Publisher Full Text

7. Seyle H: A syndrome produced by diverse nocuous agents. Nature. 1936; 138(3479): 32.

Publisher Full Text

8. Miyawaki E, Salzman C: Autonomic nervous system tests in psychiatry: 
Implications and potential uses of heart rate variability. Integrative Psychiatry. 1991; 7(1): 21-28.

Reference Source

9. Cohen $\mathrm{H}$, Kotler M, Matar MA, et al.: Power spectral analysis of heart rate variability in posttraumatic stress disorder patients. Biol Psychiatry. 1997; 41(5): 627-629. PubMed Abstract | Publisher Full Text

10. Chignon JM: [Cardiovascular pathology and panic disorder]. Can J Psychiatry. 1993; 38(2): 127-133.

PubMed Abstract | Publisher Full Text

11. Cohen $\mathrm{H}$, Benjamin J: Power spectrum analysis and cardiovascular morbidity in anxiety disorders. Auton Neurosci. 2006; 30(1-2): 1-8.

PubMed Abstract | Publisher Full Text

12. Mellman TA, Knorr BR, Pigeon WR, et al:: Heart rate variability during sleep and the early development of posttraumatic stress disorder. Biol Psychiatry. 2004; 55(9): 953-960.

PubMed Abstract | Publisher Full Text

13. Green KT, Dennis PA, Neal LC, et al.: Exploring the relationship between posttraumatic stress disorder symptoms and momentary heart rate variability. J Psychosom Res. 2016; 82: 31-4.

PubMed Abstract | Publisher Full Text | Free Full Text

14. Felitti VJ, Anda RF: The Relationship of Adverse Childhood Experiences to Adult Health, Well-being, Social Function, and Health Care. In Lanius R, Vermetten E, Pain C (eds.). The Effects of Early Life Trauma on Health and Disease: the Hidden Epidemic. Cambridge: Cambridge University Press, 2010. Publisher Full Text

15. Coid J, Petruckevltch A, Chung WS, et al:: Abusive experiences and psychiatric morbidity in women primary care attenders. Br J Psychiatry. 2003; 183(4): 332-339.

PubMed Abstract | Publisher Full Text

16. Hughes K, Lowey H, Quigg Z, et al:: Relationships between adverse childhood experiences and adult mental well-being: results from an English national household survey. BMC Public Health. 2016; 16: 222.

PubMed Abstract | Publisher Full Text | Free Full Text

17. Thayer JF, Brosschot JF: Psychosomatics and psychopathology: looking up and down from the brain. Psychoneuroendocrinology. 2005; 30(10): 1050-1058. PubMed Abstract | Publisher Full Text

18. Buchhorn R, Meint S, Willaschek C: The Impact of Early Life Stress on Growth and Cardiovascular Risk: A Possible Example for Autonomic Imprinting? PLOS One. 2016; 11(11): e0166447.

PubMed Abstract | Publisher Full Text | Free Full Text

19. Meyer PW, Müller LE, Zastrow A, et al:: Heart rate variability in patients with post-traumatic stress disorder or borderline personality disorder: relationship to early life maltreatment. J Neural Transm (Vienna). 2016; 123(9): 1107-1118

PubMed Abstract | Publisher Full Text

20. Schulz A, Vögele C: Interoception and stress. Front Psychol. 2015; 6: 993. PubMed Abstract | Publisher Full Text | Free Full Text

21. Beck AT: The evolution of the cognitive model of depression and its neurobiological correlates. Am J Psychiatry. 2008; 165(8): 969-977. PubMed Abstract | Publisher Full Text

22. Critchley HD, Nagai Y: How emotions are shaped by bodily states. Emotion Review. 2012; 4: 163-168. Publisher Full Text

23. Pollatos O, Schandry R: Emotional processing and emotional memory are modulated by interoceptive awareness. Cognition and Emotion. 2008; 22(2): 272-287.

Publisher Full Text

24. Craig AD: How do you feel--now? The anterior insula and human awareness.

Nat Rev Neurosci. 2009; 10(1): 59-70.

PubMed Abstract | Publisher Full Text
25. Strigo IA, Craig AD: Interoception, homeostatic emotions and sympathovagal balance. Philos Trans R Soc Lond B Biol Sci. 2016; 371(1708): pii: 20160010. PubMed Abstract | Publisher Full Text | Free Full Text

26. Apfel BA, Ross J, Hlavin J, et al:: Hippocampal Volume Differences in Gulf War Veterans with Current Versus Lifetime Posttraumatic Stress Disorder Symptoms. Biol Psychiatry. 2011; 69(6): 541-548. PubMed Abstract | Publisher Full Text | Free Full Text

27. Levy-Gigi E, Szabó C, Kelemen O, et al.: Association among clinical response, hippocampal volume, and FKBP5 gene expression in individuals with posttraumatic stress disorder receiving cognitive behavioral therapy. Biol Psychiatry. 2013; 74(11): 793-800

PubMed Abstract | Publisher Full Text

28. Cloitre M, Courtois CA, Ford JD, et al:: The ISTSS Expert Consensus Treatment Guidelines for Complex PTSD in Adults. 2012

Reference Source

29. Delgado-Pastor LC, Ciria LF, Blanca B, et al: Dissociation between the cognitive and interoceptive components of mindfulness in the treatment of chronic worry. J Behav Ther Ex Psychiatry. 2015; 48: 192-199. PubMed Abstract | Publisher Full Text

30. Braboszcz C, Hahusseau S, Delorme A: Meditation and Neuroscience: from basic research to clinical practice. In "Integrative Clinical Psychology, Psychiatry and Behavioral Medicine: Perspectives, Practices and Research". Editor: R. Carlstedt. Springer Publishing. 2010 Reference Source

31. Tan G, Dao TK, Farmer L, et al.: Heart rate variability (HRV) and posttraumatic stress disorder (PTSD): a pilot study. Appl Psychophysiol Biofeedback. 2011; 36(1): 27-35. PubMed Abstract | Publisher Full Text

32. Lutz A, Slagter HA, Dunne JD, et al: Attention regulation and monitoring in meditation. Trends Cogn Sci. 2008; 12(4): 163-169. PubMed Abstract | Publisher Full Text | Free Full Text

33. Foa EB, Davidson JrT, Frances A, et al:: The expert consensus guideline series. Treatment of posttraumatic stress disorder. The Expert Consensus Panels for PTSD. J Clin Psychiatry. 1999; 60 Suppl 16: 3-76. PubMed Abstract

34. Bob P, Susta M, Gregusova A, et al.: Dissociation, cognitive conflict and nonlinear patterns of heart rate dynamics in patients with unipolar depression. Prog Neuropsychopharmacol Biol Psychiatry. 2009; 33(1): 141-145. PubMed Abstract | Publisher Full Text

35. Quintana DS: Statistical considerations for reporting and planning heart rate variability case-control studies. Psychophysiology. 2017; 54(3): 344-349. PubMed Abstract | Publisher Full Text

36. Bassett $D$ : A literature review of heart rate variability in depressive and bipolar disorders. Aust N Z J Psychiatry. 2016; 50(6): 511-519.

PubMed Abstract | Publisher Full Text

37. Delorme A: R-R HRV data from Biofeedback on $\mathbf{1 0 0}$ patients (Version 6) [Data set]. Zenodo. 2020. http://www.doi.org/10.5281/zenodo.3703130

38. Van der Kolk BA: Developmental trauma disorder: Toward a rational diagnosis for children with complex trauma histories. Psychiatr Ann. 2005; 35(5): 401-408. Publisher Full Text

39. Task Force of the European Society of Cardiology the North American Society of Pacing Electrophysiology: Heart rate variability: Standards of measurement, physiological interpretation and clinical use. Task Force of the European Society of Cardiology and the North American Society of Pacing and Electrophysiology. Circulation. 1996; 93(5): 1043-1065. PubMed Abstract

40. Stemmler G, Wacker J: Personality, emotion, and individual differences in physiological responses. Biol Psychol. 2010; 84(3): 541-551. PubMed Abstract | Publisher Full Text 


\section{Open Peer Review}

\section{Current Peer Review Status:}

\section{Version 1}

Reviewer Report 16 December 2020

https://doi.org/10.5256/f1000research.22851.r75430

(C) 2020 De Witte N. This is an open access peer review report distributed under the terms of the Creative Commons Attribution License, which permits unrestricted use, distribution, and reproduction in any medium, provided the original work is properly cited.

\section{Nele De Witte}

Thomas More University of Applied Sciences, Flanders, Belgium

The current retrospective non-controlled study aimed to investigate whether the combination of a therapeutic intervention (including emotion exposure and biofeedback) influenced HR and HRV in outpatients with ACE. HR increased during the sessions but decreased from session 1 to session 2 . $\mathrm{HRV}$ at the beginning of the session increased from session 1 to session 2 . While the fact that this is a study in naturalistic circumstances can be a strength, since we are often limited to controlled research in laboratory circumstances, this also causes severe limitations in the design and interpretation of the results. Importantly, we are lacking a control group, which severely impedes interpretation. Additionally, large individual differences occurred and we would need more information about the sample to be able to interpret these. I would also be interested in seeing additional non-physiological outcome measures of the study or information about treatment effectiveness. The manuscripts requires the addition of a lot more information and the writing style and representation of the findings can be improved. However, I do think that the study could provide interesting information to the field if these improvements are made.

\section{Abstract}

"Psychiatric patients with adverse childhood experiences (ACE) tend to be dysfunctional in the interoceptive part of their emotional experience."

I would suggest to state that patients or individuals have disfunctions and not that

\section{Introduction} they are dysfunctional. This is a more respectful and correct representation.

General flow and readability: I would like to see some clarity in the definitions and presented research findings. The flow can also be improved. Subsections of the introduction appear to stand on their own. Some additional proofreading for the English language is also needed in the whole manuscript (e.g., "Interoception require the interplay between perception of body states and cognitive appraisal of these states to inform emotional experience and motivating regulatory behavior").

Specific comments:

"Heart Rate Variability reflects an individual's ability to adaptively cope with stress" 
$\mathrm{HRV}$ is a concept that can reflect many things, including emotion regulation, but also stress, general resilience, mental illness, ... (also depending on whether you are measuring resting HRV or HRV responses). So please provide some more nuance or turn the sentence around (saying that an individual's ability to adaptively cope with stress is associated with heart Rate Variability).

Please add a clear definition of HRV and ACE and add the abbreviation to the word when it is used for the first time. Subsequently, you should just use the abbreviation in the rest of the manuscript (please also do this for any other abbreviations you use). Also specify when you are talking about resting HRV or the HRV response in relation to certain stressors/paradigms/....

"Adverse childhood experiences (ACEs) are ubiquitous among the adult patient population" Could you be more specific? Are their prevalence rates available?

Please define interoception

"In fact, most psychiatric disorders are sustained by a type of interoceptive phobia" Please clarify

Neurophysiological impairments due to ACEs have been shown to be reversible Which neurophysiological impairments are you referring to here?

\section{Methods section}

Please follow a more traditional sequence in the methods section, starting with the sample. Specific comments:

Were participants instructed just notice bodily sensation or exert control over them?

\section{Consider to use "their" instead of "his/her"}

Do I understand correctly that each session of phase 2 worked with different traumatic experiences? Is it therefore possible to compare the first and last session to one another? Were you able to investigate whether a pattern emerged over different sessions or whether any change between the first and last session was possibly due to the difference in content (perhaps more difficult traumatic instances were also handled in the first session or the other way around?).

"Patient who required psychiatric medical treatment (therapist assessment) were excluded from the study"

Does this refer to receiving medication or having a diagnosis other than PTSD. It isn't entirely clear whether participants have received any formal diagnoses (if so, please specify which) or what the exact treatment context was. I was also wondering whether any underlying details on the ACE were available (how many ACEs were experienced? Severity/burden? Were there any questionnaires used?)

I noticed that the data collection section does have some details on diagnoses. Please add these to the sample section and please included how many individuals were suffering from each disorder. Could you also detail what the "clinical assessment" 
contains (is it based on a diagnostic interview, questionnaire,...).

Could you specify what individuals were exactly doing during the HRV measurement? Were they sitting, standing, lying down? Were they resting or still actively working with the traumatic experiences?

Is there any way to also report on the subjective effects of the intervention as research shows us that physiological and self-report data doesn't always agree and it would be interested to have both indicators available.

Results

Please report all results of the statistical analyses correctly (e.g., $F(2,54)=4.643, p=.014$.). You report using different HRV measurements (e.g., RMSSD, HF,...), please report which measurement you are talking about in the results. Be consistent in your terminology.

Please add significance levels to the figure and explain a bit more what it shows.

Where there any outliers that influenced results or were there non-responders?

Discussion

How do you explain that your HRV values were lower than those of the task force of the European Society of Cardiology and the North American Society of Pacing Electrophysiology?

Is there any psychophysiological data from other studies on emotional trauma recall (with or without an intervention) to compare the current data with?

You mention very large individual differences in the effect of the intervention. Does this allow us to draw general conclusions?

"However, the absence of statistical effects associated with biographical variables indicates that these types of effects are relatively unlikely."

Please explain how you see this?

"This study shows that interoception exposure therapy - combined with biofeedback - was able to increase parasympathetic influences"

This is a very strong conclusion, given that it is a non-controlled study with a lot of individual differences. Please add some nuance to this statement.

In the concluding paragraph, the authors state "HRV at rest" does this refer to the first measurement of each sessions (or both or...?).

Is the work clearly and accurately presented and does it cite the current literature? Yes

Is the study design appropriate and is the work technically sound? Partly

Are sufficient details of methods and analysis provided to allow replication by others? 
Partly

If applicable, is the statistical analysis and its interpretation appropriate?

Yes

Are all the source data underlying the results available to ensure full reproducibility? Yes

Are the conclusions drawn adequately supported by the results?

Partly

Competing Interests: No competing interests were disclosed.

Reviewer Expertise: Biofeedback, emotion regulation, HRV, e-mental health, wearables. I am not an expert in the treatment of ACE and trauma so I would recommend the relevant sections (e.g., ACE therapy with interoceptive component) to be read by an expert in this field.

I confirm that I have read this submission and believe that I have an appropriate level of expertise to confirm that it is of an acceptable scientific standard, however I have significant reservations, as outlined above.

Author Response 31 Mar 2022

Arnaud Delorme, Universite Paul Sabatier, Toulouse, France

Reviewer's suggestion: The current retrospective non-controlled study aimed to investigate whether the combination of a therapeutic intervention (including emotion exposure and biofeedback) influenced HR and HRV in outpatients with ACE. HR increased during the sessions but decreased from session 1 to session 2. HRV at the beginning of the session increased from session 1 to session 2 . While the fact that this is a study in naturalistic circumstances can be a strength, since we are often limited to controlled research in laboratory circumstances, this also causes severe limitations in the design and interpretation of the results. Importantly, we are lacking a control group, which severely impedes interpretation. Additionally, large individual differences occurred and we would need more information about the sample to be able to interpret these. I would also be interested in seeing additional non-physiological outcome measures of the study or information about treatment effectiveness. The manuscripts requires the addition of a lot more information and the writing style and representation of the findings can be improved. However, I do think that the study could provide interesting information to the field if these improvements are made.

Author's comment: We agree that the study lacks a control group. Since it is a retrospective clinical study, a control group was not possible. Still, we believe that the result of changes in patients' HRV over the course of the therapy is of scientific interest. We have clarified the method section and reorganized it. We have also worked on the writing style and remade the figure. 
Reviewer's suggestion: "Psychiatric patients with adverse childhood experiences (ACE) tend to be dysfunctional in the interoceptive part of their emotional experience." I would suggest to state that patients or individuals have dysfunctions and not that they are dysfunctional. This is a more respectful and correct representation.

Author's comment: The text has been changed.

Reviewer's suggestion: "Heart Rate Variability reflects an individual's ability to adaptively cope with stress." HRV is a concept that can reflect many things, including emotion regulation, but also stress, general resilience, mental illness, ... (also depending on whether you are measuring resting HRV or HRV responses). So please provide some more nuance or turn the sentence around (saying that an individual's ability to adaptively cope with stress is associated with heart Rate Variability).

Author's comment: We have changed the text accordingly and provided more nuance to this sentence.

Reviewer's suggestion: Please add a clear definition of HRV and ACE and add the abbreviation to the word when it is used for the first time. Subsequently, you should just use the abbreviation in the rest of the manuscript (please also do this for any other abbreviations you use). Also specify when you are talking about resting HRV or the HRV response in relation to certain stressors/paradigms/....

Author's comment: We are now clearly defining HRV and ACE and differentiate between resting HRV and HRV response. In our manuscript, we are dealing with resting HRV, but by HRV response, we mean changes in HRV over the course of the therapy.

Reviewer's suggestion: "Adverse childhood experiences (ACEs) are ubiquitous among the adult patient population". Could you be more specific? Are their prevalence rates available?

Author's comment: We have added the prevalence rate and a citation.

Reviewer's suggestion: Please define interoception

Author's comment: We are now defining interoception.

Reviewer's suggestion: "In fact, most psychiatric disorders are sustained by a type of interoceptive phobia." Please clarify.

Author's comment: We have clarified this sentence.

Reviewer's suggestion: "Neurophysiological impairments due to ACEs have been shown to be reversible." Which neurophysiological impairments are you referring to here?

Author's comment: We were referring to changes in hippocampal volume. We have clarified this sentence.

Reviewer's suggestion: Please follow a more traditional sequence in the methods section, starting with the sample. 
Author's comment: We have reorganized the method section and now start with describing our sample.

Reviewer's question: Were participants instructed just notice bodily sensation or exert control over them?

Author's comment: They were instructed just to notice them. We have clarified this point.

Reviewer's suggestion: Consider to use "their" instead of "his/her"

Author's comment: We have replaced all instances of "his/her" with "their"

Reviewer's question: Do I understand correctly that each session of phase 2 worked with different traumatic experiences? Is it therefore possible to compare the first and last session to one another? Were you able to investigate whether a pattern emerged over different sessions or whether any change between the first and last session was possibly due to the difference in content (perhaps more difficult traumatic instances were also handled in the first session or the other way around?).

Author's comment: Usually, patients' adverse experiences revolve around the same theme. The reviewer correctly mentions that there might be some heterogeneity in the ACE reported in each session. We believe this averages out across the patient population, but we have added this limitation to the discussion.

Reviewer's question: "Patient who required psychiatric medical treatment (therapist assessment) were excluded from the study." Does this refer to receiving medication or having a diagnosis other than PTSD. It isn't entirely clear whether participants have received any formal diagnoses (if so, please specify which) or what the exact treatment context was. I was also wondering whether any underlying details on the ACE were available (how many ACEs were experienced? Severity/burden? Were there any questionnaires used?)

Author's comment: This refers to patients receiving medication. Among others, questionnaires DES (Dissociative Experience Scale) and PCL-S (PTSD checklist - specific) were used with patients, although the diagnosis was based on the expertise of the clinician. We have clarified this section.

Reviewer's suggestion: I noticed that the data collection section does have some details on diagnoses. Please add these to the sample section and please include how many individuals were suffering from each disorder. Could you also detail what the "clinical assessment" contains (is it based on a diagnostic interview, questionnaire,...).

Author's comment: We have moved the section as advised. We have also clarified the clinical diagnosis.

Reviewer's suggestion: Could you specify what individuals were exactly doing during the HRV measurement? Were they sitting, standing, lying down? Were they resting or still 
actively working with the traumatic experiences?

Author's comment: All patients were sitting at rest. We have clarified this point.

Reviewer's suggestion: Is there any way to also report on the subjective effects of the intervention as research shows us that physiological and self-report data doesn't always agree and it would be interesting to have both indicators available.

Author's comment: this is an interesting comment. The current data collection scheme includes the subjective assessment of both the patient and the therapist, who, after a given number of sessions, which might be different for each patient, agreed that the therapy was complete.

Reviewer's suggestion: Please report all results of the statistical analyses correctly (e.g., $F$ (2, $54)=4.643, p=.014$.). You report using different HRV measurements (e.g., RMSSD, HF, ...), please report which measurement you are talking about in the results. Be consistent in your terminology.

Author's comment: We have changed statistical reporting. We have also clarified the measures being reported.

Reviewer's suggestion: Please add significance levels to the figure and explain a bit more what it shows.

Author's comment: We have added significance to the figure and additional details to its caption.

Reviewer's question: Where there any outliers that influenced results or were there nonresponders?

Author's comment: This is a detailed analysis we did not perform. We can expect that there were non-responders and outliers, although based on the age of the data, it would take considerable effort to find the number of non-responders, and we do not think it would necessarily be informative - observing no response does not imply that the patient did not respond to the treatment. It might be possible that for a given patient with no increase in $\mathrm{HRV}$, the coupling of emotional traumas with HRV is weaker. ANOVA has poor resistance to outliers, so if there were outliers, their presence was not prominent enough to invalidate our results. We have added a comment to the discussion.

Reviewer's question: How do you explain that your HRV values were lower than those of the task force of the European Society of Cardiology and the North American Society of Pacing Electrophysiology?

Author's comment: Low HRV is associated with stress, and the data from the European Society of Cardiology and the North American Society of Pacing Electrophysiology were for control participants. It is to be expected that HRV of patients, in general, would be lower than control participants. We have added that interpretation to the manuscript. 
Reviewer's question: Is there any psychophysiological data from other studies on emotional trauma recall (with or without an intervention) to compare the current data with?

Author's comment: We are not aware of studies on emotional trauma recall using HRV that report the measure that we are reporting for detailed comparisons. We have added a citation to a recent study on the subject, but the data is not available. By contrast, we have made our data available. This study is consistent with decreased HRV in the population of ACE patients.

Reviewer's question: You mention very large individual differences in the effect of the intervention. Does this allow us to draw general conclusions?

Author's comment: HRV is a noisy physiological measure. Large differences in HRV are common in the population, with centenarians having HRV comparable to 20-year-old individuals (despite the well-established decrease in HRV with age). Therefore, large differences in HRV response to treatment are also expected. The origin of HRV interindividual differences is unknown to our knowledge.

Reviewer's question: "However, the absence of statistical effects associated with biographical variables indicates that these types of effects are relatively unlikely." Please explain how you see this?

Author's comment: We have rephrased that section to clarify this statement.

Reviewer's suggestion: "This study shows that interoception exposure therapy - combined with biofeedback - was able to increase parasympathetic influences" This is a very strong conclusion, given that it is a non-controlled study with a lot of individual differences. Please add some nuance to this statement.

Author's comment: We have added nuance to that statement.

Reviewer's question: In the concluding paragraph, the authors state "HRV at rest" does this refer to the first measurement of each sessions (or both or...?)

Author's comment: This refers to both measurements. We have clarified this in the method section.

Competing Interests: No competing interests were disclosed. 
(C) 2020 Şar V. This is an open access peer review report distributed under the terms of the Creative Commons Attribution License, which permits unrestricted use, distribution, and reproduction in any medium, provided the original work is properly cited.

\section{Vedat Şar}

Department of Psychiatry, Koç University School of Medicine, Istanbul, Turkey

This study is based on the hypothesis that psychiatric patients with adverse childhood experiences (ACE) tend to be dysfunctional in the interoceptive dimension of the emotional experience which is characterized by diminished sympathovagal balance. A sufficient number of participants was subjected to exposure related to their ACE while taking the heart rate variability (HRV) to monitor the effect of the intervention. The latter covered also biofeedback sessions. A significant increase in HRV and decrease in heart rate were obtained at the end. The strength of this study is the hypothesis which is based on a firm theoretical basis and the transdiagnostic approach. The weakness of the study is lack of long-term follow up.

This is a useful and well written study. To strengthen the paper, the authors should also address polyvagal theory (Porges) shortly to enrich the paper. Another subject to address is the place of HRV in the literature of PTSD that possible differences between effects of adverse childhood experiences and adult types of traumatization on HRV is dealt with.

Is the work clearly and accurately presented and does it cite the current literature? Yes

Is the study design appropriate and is the work technically sound?

Yes

Are sufficient details of methods and analysis provided to allow replication by others? Yes

If applicable, is the statistical analysis and its interpretation appropriate? Yes

Are all the source data underlying the results available to ensure full reproducibility? Yes

Are the conclusions drawn adequately supported by the results? Yes

Competing Interests: No competing interests were disclosed.

Reviewer Expertise: PTSD, dissociative disorders, borderline personality disorder, functional neurological disorders, epidemiology, depression

I confirm that I have read this submission and believe that I have an appropriate level of expertise to confirm that it is of an acceptable scientific standard. 
The benefits of publishing with F1000Research:

- Your article is published within days, with no editorial bias

- You can publish traditional articles, null/negative results, case reports, data notes and more

- The peer review process is transparent and collaborative

- Your article is indexed in PubMed after passing peer review

- Dedicated customer support at every stage

For pre-submission enquiries, contact research@f1000.com 